Recibido: $19-\mid-2020$

Aceptado:

$16-1 \mid 1-2020$

Publicado en línea:

23-III-2020
Percepción estética de la sonrisa según variación de la posición vertical y de la angulación del incisivo central superior en tres regiones del Perú

\section{Esthetic Perception of the Smile According to Variation of the Vertical Position and the Angulation of the Upper Central Incisor in Three Regions of Peru}

Luis Angel García-Gonzales DDS1; Teresa Angélica Evaristo-Chiyong DDS,MSc,PhD²

1. Egresado, Maestría en estomatología. Universidad Científica del Sur, Lima, Perú.

2. Maestría en estomatología. Universidad Científica del Sur, Lima, Perú

Autor para correspondencia: Dr. Luis Angel García-Gonzales - luis-dent@hotmail.com

RESUMEN: Objetivo: Evaluar la percepción estética de la sonrisa según variación de la posición vertical y de la angulación del incisivo central superior (ICS) en estudiantes de odontología (E0) y personas comunes (PC) de las tres regiones del Perú. Materiales y Métodos: Diseño descriptivo, transversal. La muestra fueron 462 adultos, divididos en 77 para cada subgrupo de E0 y PC de las regiones Lima (Costa), Junín (Sierra) y Loreto (Selva). Mediante el programa Photoshop se modificó una fotografía de sonrisa de mujer variando la posición vertical y angulación del ICS, obteniéndose imágenes que fueron evaluadas mediante la escala visual análoga. Resultados: Las PC calificaron mejor que los E0 en la mayoría de las categorías $(p<0,001)$. La sonrisa mejor valorada por E0 en posición vertical fue a $1 \mathrm{~mm}$ y $2 \mathrm{~mm}$; y para angulación $0^{\circ}$, mientras que para PC la de $1 \mathrm{~mm}$ y de $0^{\circ}$ respectivamente $(p<0,05)$. Comparando por regiones los E0 de Lima dieron la menor calificación para $0^{\circ}$ con 52,63 y los de Junín la mayor para $4^{\circ}$ con 45,90 . Las PC de Loreto registraron el menor puntaje para las categorías de posición vertical $(p<0,001)$, mientras que para angulación en Junín se calificó con un menor puntaje que en Loreto para $-6^{\circ}$ y $0^{\circ}$; y para $4^{\circ}$ Lima brindó la menor calificación $(p<0,001)$. Conclusiones: La percepción estética de la sonrisa es afectada por la variación de posición vertical y angulación del ICS en personas comunes y estudiantes de odontología en las tres regiones del Perú.

PALABRAS CLAVE: Percepción, Estética, Posición vertical, Angulación mesiodistal, Sonrisa, Perú. 
ABSTRACT: Objective: To evaluate the esthetic perception of the smile according to the variation of the vertical position and the angulation of the upper central incisor (UPI) by dental students (DS) and common people (CP) of three regions of Peru. Materials and Methods: Descriptive cross-sectional design. The sample was 462 adults, divided into 77 for each subgroup of DS and CP of the Lima (Coast), Junín (Highlands) and Loreto (Rainforest) regions. Using the Photoshop ${ }^{\circledR}$ software, a photograph of a woman's smile was modified by varying the vertical position and angulation of the UPI, obtaining images that were evaluated using the analog visual scale. Results: The $C P$ rated better than DS in most categories $(p<0.001)$. The smile best valued by DS in a vertical position was $1 \mathrm{~mm}$ and $2 \mathrm{~mm}$; and for angulation $0^{\circ}$, while for $C P 1 \mathrm{~mm}$ and $0^{\circ}$ respectively $(p<0.05)$. Comparing by regions, the DS of Lima gave the lowest rating for $0^{\circ}$ with 52.63 and those of Junín the highest for $4^{\circ}$ with 45.90. The PC of Loreto registered the lowest score for the vertical position categories $(p<0.001)$, while for angulation in Junín it was rated with a lower score than in Loreto for $-6^{\circ}$ and 0 $\circ$; and for $4^{\circ}$ Lima provided the lowest rating $(p<0.001)$. Conclusions: The esthetic perception of the smile is affected by the variation of vertical position and angulation of the ICS in common people and dental students in the three regions of Peru.

KEYWORDS: Perception, Esthetics, Vertical position, Mesiodistal angulation, Smile, Peru.

\section{INTRODUCCIÓN}

En los últimos años la búsqueda de una adecuada estética facial y dental, ha tomado mayor importancia para los pacientes, siendo la sonrisa un elemento primordial en ello. Actualmente es frecuente someterse a diferentes tratamientos estéticos y cosméticos en las diversas especialidades médicas y odontológicas, sin embargo, no existe un consenso entre ortodoncistas, dentistas, estudiantes de odontología, pacientes y personas comunes en lo que se considera una sonrisa estética (1-5), esto se debe posiblemente a la naturaleza subjetiva de la sonrisa y a que durante muchos años solo se tomó en cuenta la opinión de algunos autores y no de una literatura basada en evidencias $(6,7)$. Es importante mencionar que en la planificación del tratamiento ortodóntico y en la finalización, el objetivo principal ha sido lograr una oclusión estable con una adecuada función y esto no necesariamente coincide con una sonrisa estética en el sector anterior (8-10).
La percepción estética de un individuo se encuentra influenciada por factores como edad, nivel educativo, estado social, diferencias culturales y origen étnico, así como difiere según poblaciones, país, región o continente (11-14). Medios de comunicación incluyendo televisión radio, publicidad, películas, revistas e internet también juegan un papel importante en la cultura moderna (15).

Existe múltiples factores relacionados a la estética de la sonrisa (16) que pueden dividirse en microestética, estética gingival, macroestética y estética facial (17), de los cuales se puede mencionar que las líneas de sonrisas rectas 0 invertidas se consideran menos atractivas $(7,18)$, mientras que las líneas convexas son consideradas más bellas y jóvenes (19); tomando suma importancia la posición vertical del incisivo central superior(ICS) $(7-8,20,21)$ y su relación con el borde incisal del incisivos lateral superior(ILS) $(8,22)$ para que una sonrisa sea considerada atractiva o estética. 
En relación al efecto de la angulación mesiodistal del ICS en el atractivo de la sonrisa aún no está del todo claro (23), pero se sabe que tiene influencia en el punto de contacto entre incisivos considerado un factor estético $(17,24)$. El grado de angulación determina la cantidad de espacio mesiodistal que utilizan estos dientes, lo cual afecta la cantidad de espacio disponible para los dientes posteriores (25).

El propósito de este estudio fue evaluar la percepción estética de la sonrisa según variación de la posición vertical y angulación del incisivo central superior de estudiantes de odontología y personas comunes en tres regiones del Perú en donde no existen antecedentes previos de este comportamiento que es muy importante para establecer objetivos estéticos comunes a fin de que todas las personas relacionadas queden conformes y el tratamiento ortodóntico sea considerado exitoso.

\section{MATERIALES Y MÉTODOS}

\section{DISEÑO DEL ESTUDIO}

El presente estudio fue aprobado por el Comité Institucional de Ética en Investigación de la Universidad Científica del Sur con número de registro 252-2018-POS99, asimismo los participantes firmaron voluntariamente el consentimiento para ser considerados parte de la investigación. El diseño fue descriptivo transversal, a partir de los resultados de un estudio piloto se utilizó la fórmula de comparación de medias con un nivel de confianza de $95 \%$, poder estadístico de $80 \%$, varianza de 167,30 y precisión de 6 , determinándose un tamaño de muestra de 77 para cada subgrupo de estudiantes de odontología y personas comunes en cada región: Lima (Costa), Junín (Sierra) y Loreto (Selva), haciendo un total de 462 adultos evaluados. Se tuvo como criterio para la elección de la muestra que la edad de los participantes estuviera entre 18 y 60 años y que tuvieran como residencia la región donde se realizaba la encuesta, que los estudiantes de odontología se encontraran cursando entre el 5to al 10 ciclo y que las personas comunes no se encontraran recibiendo algún tratamiento dental considerado estético.

Los estudiantes fueron seleccionados de manera aleatoria simple en las facultades de odontología de la Universidad Científica del Sur (Lima), Universidad Privada de Huancayo Franklin Roosevelt (Junín) y Universidad Nacional de la Amazonia Peruana (Selva) contando con la colaboración de los docentes responsables de las distintas asignaturas quiénes dieron un espacio de tiempo para el desarrollo del trabajo. Las personas comunes fueron los acompañantes de pacientes o personas que acudieron a las clínicas odontológicas para otro tipo de tratamiento que no fuese estético en cada una de las universidades de dónde se reclutaron a los estudiantes y que cumplieron con los criterios de selección. Ellos fueron seleccionados a manera de casos consecutivos hasta completar el tamaño requerido.

\section{ELECCIÓN Y TOMA DE FOTOGRAFÍA}

Una fotografía frontal de sonrisa de una mujer de 28 años fue seleccionada para el estudio, la cual era considerada atractiva de acuerdo algunos principios descritos en la literatura (6,8,22): una adecuada proporción ancho/longitud en la zona estética, $1 \mathrm{~mm}$ de exposición gingival a nivel del ICS, contorno gingival del canino coincidentes con el ICS y $0.5 \mathrm{~mm}$ por encima del ILS, línea media superior e inferior coincidentes, borde incisal del ICS $1 \mathrm{~mm}$ más extruido que el borde del ILS y longitud de la corona del ICS levemente más larga que el canino superior. La fotografía fue tomada con una cámara digital Nikon D5200 en modo automático con ring flash en la posición estética recomendada por Bass (26), posición natural de cabeza asistida por un operador. 


\section{EDICIÓN DE FOTOS}

La foto seleccionada y todas las ediciones fueron realizadas por el mismo operador usando el Programa Adobe Photoshop CC 2018. La foto inicial fue manipulada y reflejada para lograr una imagen con una simetría de derecha a izquierda; se editó para ajustar el color, el brillo, el contraste y para eliminar cualquier decoloración en los labios y la piel. También se recortó, eliminándose la nariz y el mentón y así reducir el número de variables que pudieran distraer al evaluador. Luego fue expandida y comprimida con las herramientas del programa, utilizando al ICS derecho como referencia, con el fin de obtener la medida real de la sonrisa de la paciente, de esta manera cada milímetro medido en la imagen digital e impresa fue equivalente al medido clínicamente. La validez de la imagen final fue determinada por tres especialistas en ortodoncia y un especialista en estética restauradora.

\section{IMÁGENES CON VARIACIÓN DE LA POSICIÓN VERTICAL}

Se utilizó como referencia la línea imaginaria formada por los bordes incisales de los ILS para extruir e intruir $1 \mathrm{~mm}$ respectivamente los ICS, obteniéndose así imágenes que permitieron evaluar la posición vertical en $0 \mathrm{~mm}, 1 \mathrm{~mm}$ y $2 \mathrm{~mm}$ (Figura 1A, B y C)

\section{IMÁGENES CON VARIACIÓN DE LA ANGULACIÓN MESIODISTAL}

La referencia utilizada fue el eje facial de la corona clínica del incisivo central superior, la cual fue girada en sentido horario y antihorario teniendo como centro de rotación el margen gingival, obteniéndose así imágenes con una diferencia en grados con relación al eje inicial. La angulación mesiodistal del incisivo central superior fue evaluada a $-6^{\circ}, 0^{\circ}$ y $4^{\circ}$ (Figura $1 D, B$ y E).

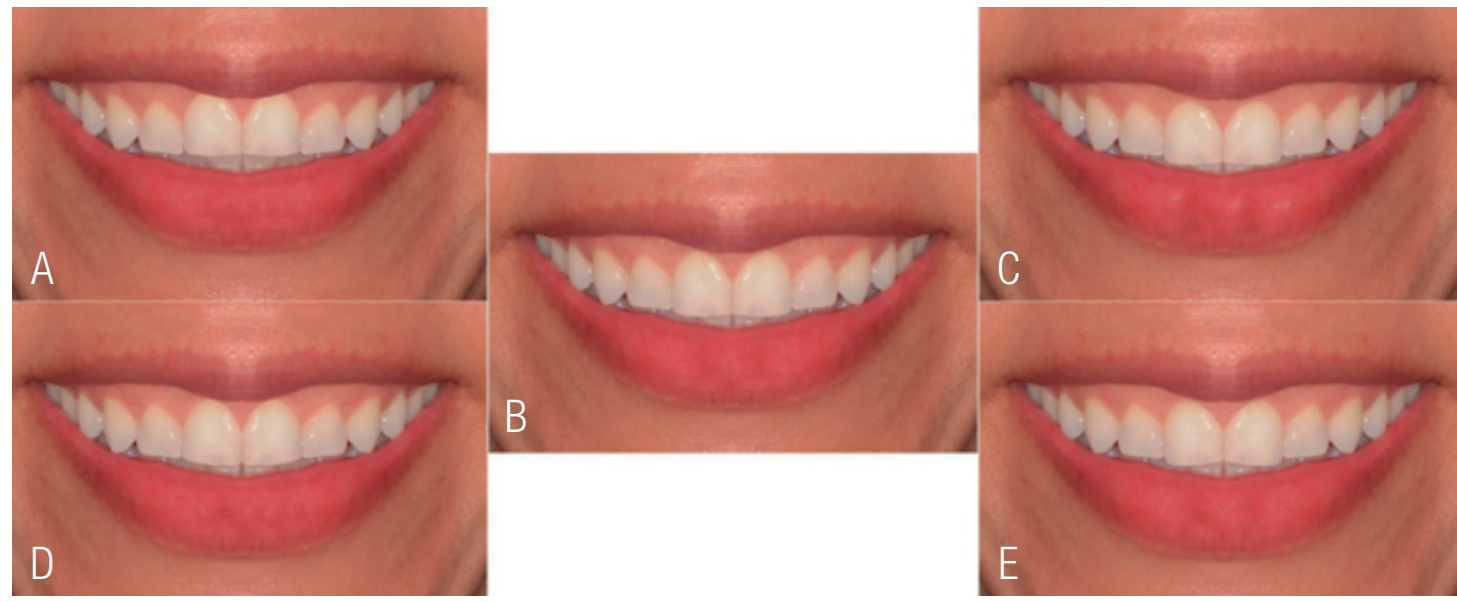

Figura 1. Imágenes con variación de la posición vertical y la angulación del incisivo central superior. $A=0 \mathrm{~mm}, B=1 \mathrm{~mm}$ y $0^{\circ}, \mathrm{C}=2 \mathrm{~mm}, \mathrm{D}=-6^{\circ}, \mathrm{E}=4^{\circ}$.

ÁLBUM FOTOGRÁFICO, CUESTIONARIO Y ESCALAS VAS

Los archivos digitales de las imágenes finales presentaron una resolución de 300 dpi y un tamaño de $(90,8 \mathrm{~mm} \times 55,9 \mathrm{~mm})$, las cuales fueron impresas en papel fotográfico para la confección de un álbum en donde se presentó una imagen por página, primero la secuencia de la posición vertical y luego la de angulación mesiodistal. El orden en que se mostraron las mismas fue obtenido de manera aleatoria simple para cada una de las 
variables, siendo el siguiente $0 \mathrm{~mm}, 2 \mathrm{~mm}, 1 \mathrm{~mm}$, $-4^{\circ}, 6^{\circ}$ y $0^{\circ}$.

Se utilizó la escala visual analógica (VAS) de $100 \mathrm{~mm}$ para la evaluación de cada imagen para darle una mayor sensibilidad a la medición de la variable. El lado izquierdo de la escala represento el menos atractivo $(0 \mathrm{~mm})$ mientras que el derecho el más atractivo $(100 \mathrm{~mm})$, se colocó una línea central como referencia. Para controlar el sesgo se instruyó a todos los participantes, se les mostró las imágenes en un mismo orden y cada una de ellas durante 30 segundos, luego de observar una imagen debían calificar marcando con una línea perpendicular en la escala VAS antes de continuar con la siguiente, no se permitió la reevaluación a fin de evitar comparaciones entre ellas. Asimismo, para limitar el posible cansancio visual se decidió evaluar sólo tres posiciones en cada una de las variables. Todas las marcas realizadas en las escalas fueron medidas con un Vernier digital electrónico por el mismo investigador.

Se utilizó el Coeficiente de correlación intraclase para determinar la consistencia interna del instrumento, obteniéndose un coeficiente Alfa de Cronbach de 0.574 .

\section{ANÁLISIS ESTADÍSTICO}

Los datos fueron sometidos a análisis estadísticos con el software SPSS 24. Se reportó la estadística descriptiva a través de media y desviación estándar. La comparación entre personas comunes y estudiantes de odontología se realizó con la prueba U de Mann Whitney o T student, para comparar entre regiones se utilizó ANOVA o Kruskall wallis todas ellos dependiendo del cumplimiento de los supuestos estadísticos. Se aplicó la ecuación de estimación generalizada para evaluar el efecto del tipo de observador, región a la que pertenecía y la variación de la posición vertical y la angulación en la percepción estética. El nivel de significancia fue establecido al $5 \%$.

\section{RESULTADOS}

La muestra estuvo conformada por 462 sujetos siendo 243 de sexo femenino y 219 de sexo masculino. La edad en personas comunes fue en promedio $26,45 \pm 9,61$ mientras que en los estudiantes fue $24,85 \pm 5,89$. La mediana de edad en ambos grupos fue 23 y 22 años respectivamente (Tabla 1).

Al comparar la percepción estética según el tipo de evaluador, se encontró que para la variación de la posición vertical del ICS solo hubo diferencia significativa en la imagen de $1 \mathrm{~mm}$, con un mayor puntaje otorgado por las personas comunes que los estudiantes de Odontología, las comparaciones dentro de cada grupo muestran como imagen con mayor puntaje a la de $1 \mathrm{~mm}$ y $2 \mathrm{~mm}$ para estudiantes de odontología y de $1 \mathrm{~mm}$ para personas comunes. En relación a la angulación del ICS se encontró diferencias estadísticas para todas las imágenes $\left(-6^{\circ}, 0^{\circ}\right.$ y $\left.4^{\circ}\right)$ con un mayor puntaje otorgado por las personas comunes, las comparaciones en ambos grupos muestran que la imagen con mayor puntaje fue la de $0^{\circ}$ (Tabla 2).

Cuando se compara la percepción estética según la posición vertical del ICS en estudiantes de Odontología por región, no se encuentra diferencia estadística entre ellas y dentro de cada grupo se encuentra que para Lima la imagen con mayor puntuación fue la de $2 \mathrm{~mm}$ y que para Loreto la de menor puntuación fue la de $0 \mathrm{~mm}$. Mientras al comparar personas comunes por región se encontró diferencias estadísticas para todas las imágenes obteniendo una menor puntuación las que proceden de Loreto, dentro de cada grupo se observa que la imagen con mayor valoración para las 3 regiones fue la de $1 \mathrm{~mm}$, pero en el caso de 
Lima esta no presenta diferencia estadística con la de $2 \mathrm{~mm}$ (Tabla 3).

Al variar la posición del ICS aumenta la calificación de la percepción estética de la sonrisa, siendo esta mayor en la posición a $1 \mathrm{~mm}$ en comparación a $0 \mathrm{~mm}$. Las personas comunes califican la sonrisa con una puntuación mayor en comparación a los estudiantes de odontología, mientras que las personas de la Selva tienen una percepción menor de la estética en comparación a la de la Sierra y de la Costa (Tabla 4).

Al comparar la percepción estética de la sonrisa según la angulación del ICS de estudiantes de odontología por región se encontró diferencia en la imagen de $0^{\circ}$ con un mayor puntaje otorgado por la región de Junín sobre la de Lima y en el caso de la imagen de $4^{\circ}$ la región Junín también brindo un mayor puntaje que Lima y Loreto, en la comparación dentro de cada grupo se encontró que la imagen con mayor valoración fue la de $0^{\circ}$ para todas las regiones. Comparando la percepción estética de la angulación de personas comunes por regiones se encontró diferencias significativas, para las imágenes $-6^{\circ}$ y $0^{\circ}$ Junín valoro con mayor puntaje y para $4^{\circ}$ Lima otorgo el menor puntaje, al comparar dentro de cada grupo se encontró que la imagen de $0^{\circ}$ fue la mejor valorada para las tres regiones, pero en la región de Junín no presento diferencia estadística con la de $-6^{\circ}$ (Tabla 5).

La angulación del ICS al ser aumentada a $4^{\circ}$ o disminuida a $-6^{\circ}$, disminuye la percepción estética en comparación a cero grados de angulación. La persona común en comparación al estudiante de odontología, así como las de la selva y la de sierra perciben de manera más estética la sonrisa ante los cambios de la angulación que las de la costa (Tabla 6).

Tabla 1. Distribución de la edad según tipo de observador.

\begin{tabular}{cccccc}
\hline & \multicolumn{5}{c}{ Edad en años } \\
\cline { 2 - 6 } & Media & DE & Primer cuartil & Segundo cuartil & Tercer cuartil \\
\hline Estudiante & 24,85 & 5,89 & 21 & 23 & 27 \\
Persona común & 26,45 & 9,61 & 20 & 22 & 31 \\
\hline
\end{tabular}

Tabla 2. Percepción de la sonrisa de acuerdo a la posición vertical y angulación del ICS según tipo de observador

\begin{tabular}{|c|c|c|c|c|c|}
\hline \multirow{3}{*}{ Variables } & \multicolumn{4}{|c|}{ Tipo de observador } & \multirow[b]{3}{*}{$\mathbf{p}$} \\
\hline & \multicolumn{2}{|c|}{ Estudiante de odontología } & \multicolumn{2}{|c|}{ Persona común } & \\
\hline & Media & DE & Media & $\mathrm{DE}$ & \\
\hline \multicolumn{6}{|l|}{ Posición } \\
\hline $0 \mathrm{~mm}$ & $46,58^{a}$ & 20,06 & $48,80^{a}$ & 18,54 & $0,247^{*}$ \\
\hline $1 \mathrm{~mm}$ & $51,37^{b}$ & 16,42 & $57,97 \mathrm{~b}$ & 16,34 & $<0,001^{*}$ \\
\hline $2 \mathrm{~mm}$ & $52,63^{b}$ & 17,38 & $54,65 c$ & 17,74 & $0,217+$ \\
\hline \multicolumn{6}{|l|}{ Angulación } \\
\hline$-6^{\circ}$ & $50,43^{a}$ & 18,37 & $57,44^{\mathrm{a}}$ & 17,89 & $<0,001^{*}$ \\
\hline $0^{\circ}$ & $57,52^{b}$ & 18,34 & $63,52^{b}$ & 19,61 & $<0,001^{*}$ \\
\hline $4^{\circ}$ & $39,46^{c}$ & 18,92 & $48,66^{\mathrm{c}}$ & 19,34 & $<0,001^{\star}$ \\
\hline
\end{tabular}

* $\mathrm{U}$ de Mann Whitney, $+\mathrm{T}$ de Student.

Letras distintas: grupos distintos dentro de cada tipo de observador en cada una de las variables. 
Tabla 3. Percepción de la sonrisa de acuerdo a la posición del ICS según tipo de observador y región.

\begin{tabular}{|c|c|c|c|c|c|c|c|}
\hline \multirow[b]{3}{*}{$\begin{array}{l}\text { Obsevador/ } \\
\text { Posición }\end{array}$} & \multicolumn{6}{|c|}{ Región } & \multirow[b]{3}{*}{$\mathbf{p}$} \\
\hline & \multicolumn{2}{|c|}{ Lima } & \multicolumn{2}{|c|}{ Junín } & \multicolumn{2}{|c|}{ Loreto } & \\
\hline & Media & $\mathrm{DE}$ & Media & $\mathrm{DE}$ & Media & DE & \\
\hline \multicolumn{8}{|l|}{ Estudiante } \\
\hline $0 \mathrm{~mm}$ & $46,96 a$ & 18,97 & $48,49 a$ & 23,65 & $44,29 a$ & 17,02 & $0,421^{*}$ \\
\hline $1 \mathrm{~mm}$ & $51,31 \mathrm{a}$ & 16,16 & $52,13 a$ & 17,63 & $50,66 b$ & 15,59 & $0,858+$ \\
\hline $2 \mathrm{~mm}$ & $54,82 b$ & 16,04 & $51,69 a$ & 19,60 & $51,37 b$ & 16,29 & $0,396+$ \\
\hline $\mathrm{P}$ & $<0,001 \ddagger$ & & $0,131 \ddagger$ & & $<0,001 \ddagger$ & & \\
\hline \multicolumn{8}{|l|}{ Persona común } \\
\hline $0 \mathrm{~mm}$ & $50,02 \mathrm{a} 1$ & 17,02 & $53,51 \mathrm{a} 1$ & 20,07 & $42,86 a 2$ & 16,98 & $0,001^{*}$ \\
\hline $1 \mathrm{~mm}$ & $61,52 b 1$ & 14,64 & $59,70 b 1$ & 17,16 & $52,67 \mathrm{~b} 2$ & 15,97 & $<0,001^{*}$ \\
\hline $2 \mathrm{~mm}$ & $60,94 b 1$ & 16,95 & $55,88 \mathrm{a} 1$ & 16,90 & $47,12 \mathrm{c} 2$ & 16,76 & $<0,001+$ \\
\hline$p$ & $<0,001 \S$ & & $<0,001 \ddagger$ & & $<0,001 \S$ & & \\
\hline
\end{tabular}

* Kruskall Wallis, +Anova de un factor: números distintos indican grupos distintos entre regiones dentro de cada posición evaluada. ‡ Anova medidas repetidas, § Friedman: letras distintas: indican grupos distintos entre cada una de las posiciones evaluadas.

Tabla 4. Efecto de la variación de la posición, tipo de observador y región en la percepción estética de la sonrisa.

\begin{tabular}{lcccc}
\hline \multicolumn{1}{c}{ Variables } & Coef B & \multicolumn{1}{c}{ IC 95\% } & $\mathbf{p}^{\star}$ \\
\hline Posición & & & & \\
A 2mm & 5,95 & 4,49 & 7,41 & $<0,001$ \\
A 1mm & 6,98 & 5,52 & 8,44 & $<0,001$ \\
Omm & Referencia & & & \\
Observador & & & & \\
Persona común & 3,61 & 0,81 & 6,42 & 0,012 \\
Estudiante & Referencia & & & \\
Región & & & & \\
Selva (Loreto) & $-6,10$ & $-9,22$ & $-2,98$ & 0,000 \\
Sierra (Junín) & $-0,70$ & $-4,22$ & 2,83 & \\
Costa (Lima) & Referencia & & & \\
\hline
\end{tabular}

*Ecuación de estimación generalizada. 
Tabla 5. Percepción de la sonrisa de acuerdo a la angulación del ICS según tipo de observador y región.

\begin{tabular}{|c|c|c|c|c|c|c|c|}
\hline \multirow[b]{3}{*}{$\begin{array}{l}\text { Obsevador/ } \\
\text { Posición }\end{array}$} & \multicolumn{6}{|c|}{ Región } & \multirow[b]{3}{*}{$\mathbf{p}$} \\
\hline & \multicolumn{2}{|c|}{ Lima } & \multicolumn{2}{|c|}{ Junín } & \multicolumn{2}{|c|}{ Loreto } & \\
\hline & Media & DE & Media & $\mathrm{DE}$ & Media & DE & \\
\hline \multicolumn{8}{|l|}{ Estudiante } \\
\hline$-6^{\circ}$ & $47,31^{a}$ & 20,82 & $53,07^{a}$ & 18,22 & $50,90^{a}$ & 15,45 & $0,203^{*}$ \\
\hline $0^{\circ}$ & $52,63^{a 1}$ & 18,67 & $60,17^{\mathrm{b} 1}, 2$ & 18,21 & $59,77^{\text {b2 }}$ & 17,35 & $0,016^{*}$ \\
\hline $4^{\circ}$ & $34,90^{b 1}$ & 19,16 & $45,90^{2}$ & 20,30 & $37,59^{-1}$ & 15,42 & $0,001+$ \\
\hline$p$ & $<0,001 \S$ & & $<0,001 \S$ & & $<0,001 \ddagger$ & & \\
\hline \multicolumn{8}{|l|}{ Persona común } \\
\hline$-6^{\circ}$ & $57,43^{\mathrm{a} 1}, 2$ & 15,02 & $61,87^{\mathrm{ab} 1}$ & 21,56 & $53,03^{\mathrm{a} 2}$ & 15,43 & $0,001^{*}$ \\
\hline $0^{\circ}$ & $64,76^{\mathrm{b} 1},{ }^{2}$ & 18,69 & $67,06^{a_{1}}$ & 19,54 & $58,75^{\text {b } 2}$ & 19,89 & $0,014^{*}$ \\
\hline $4^{\circ}$ & $36,57^{\mathrm{cl}}$ & 13,41 & $58,53^{\mathrm{b} 2}$ & 19,89 & $50,89^{a} 2$ & 17,46 & $<0,001^{*}$ \\
\hline$p$ & $<0,001 \S$ & & $<0,001 \ddagger$ & & $<0,001 \S$ & & \\
\hline
\end{tabular}

*Kruskall Wallis +Anova de un factor, números distintos indican grupos distintos entre regiones dentro de cada posición evaluada. ‡ Anova de medidas repetidas, § Friedman: letras distintas indican grupos distintos entre cada una de las posiciones evaluadas dentro de cada región.

Tabla 6. Efecto de la variación de angulación, tipo de observador y región en la percepción estética de la sonrisa.

\begin{tabular}{lcccc}
\hline \multicolumn{1}{c}{ Variables } & Coef $\mathbf{B}$ & \multicolumn{1}{c}{ IC 95\% } & $\mathbf{p}^{*}$ \\
\hline Angulación & & & & \\
A $4^{\circ}$ & $-16,46$ & $-18,25$ & $-14,67$ & $<0,001$ \\
A $-^{\circ}$ & $-6,59$ & $-8,16$ & $-5,02$ & $<0,001$ \\
A $0^{\circ}$ & Referencia & & & \\
Observador & & & & \\
Persona común & 7,40 & 4,66 & 10,15 & $<0,001$ \\
Estudiante & Referencia & & & \\
Región & & & & \\
Selva (Loreto) & 2,89 & $-0,23$ & 6,00 & \\
Sierra (Junín) & 8,83 & 5,39 & 12,28 & $<0,001$ \\
Costa (Lima) & Referencia & & & \\
\hline
\end{tabular}

*Ecuación de estimación generalizada. 
DISCUSIÓN

En la percepción estética de la sonrisa el ICS es considerado una llave determinante y un aspecto de suma importancia $(8,20)$, ya que influye en diferentes procedimientos odontológicos como restauraciones estéticas, prótesis dental fija y posicionamiento de brackets. En el presente estudio se ha evaluado su posición vertical y angulación mesiodistal desde una vista frontal con el objetivo de obtener mayor información del comportamiento de estas variables, mientras que la inclinación vestibulolingual no ha sido considerada debido a que es un factor estudiado y tiene mayor relevancia desde una vista de perfil $(27,28)$, sin embargo Almutairi et al. (29) reportaron previamente diferencias entre una sonrisa con biprotrusion dentaria e incisivos vestibularizados y otra post tratamiento desde una vista frontal y de $45^{\circ}$, por lo cual se considera que es un factor que podría ser evaluado en futuros estudios.

En la metodología para identificar la posición vertical ideal existen diferentes implicancias en los bordes incisales y márgenes gingivales, para efectos de este estudio se siguió el mismo método sugerido por Machado et al. (8) manteniendo la anatomía de los dientes. De esta manera cuando los ICS fueron intruidos la cantidad de exposición gingival disminuyó y por el contrario cuando fueron extruidos ésta aumentó, evitando alterar el tamaño del ICS y la proporción ancho/longitud que podrían influir en la percepción estética; asimismo se utilizó como referencia la posición vertical considerada ideal con una diferencia de $1 \mathrm{~mm}$ entre el borde incisal del ICS e ILS mencionada por el mismo autor en sus estudios previos (7$8,22)$. También fue evaluada la influencia de la angulación mesiodistal del ICS con la finalidad de comprender mejor la estética de la sonrisa, ya que existen pocos antecedentes previos de esta variable (23).
Con el objetivo de evitar distracciones con otras características faciales se decidió utilizar fotografías del tercio inferior, estudios previos han demostrado que no existe diferencia significativa al evaluar la sonrisa con fotografías de toda la cara $(21,22)$.

El instrumento de evaluación utilizado fue la escala VAS la cual brinda un puntaje confiable (30) y es un método de uso común para nociones subjetivas como el dolor, atractivo y ansiedad en investigaciones de ciencias de la salud; aunque existe la tendencia de algunos evaluadores de no utilizar toda la escala debido a que tienen temor de dar una calificación alta a una imagen, en caso de que la siguiente les parezca más estética (31), para compensar esto y limitar el posible cansancio visual se decidió evaluar sólo tres posiciones en cada una de las variables estudiadas, a diferencia de otros estudios en dónde evaluaron mayor cantidad (1-5,8-10).

La edad promedio de los participantes estudiantes y personas comunes es el reflejo de una población principalmente adulto joven, donde los patrones estéticos pueden verse mucho más influenciados por condiciones estacionales y coyunturales como la moda. Resultados de estudios previos sugieren que la percepción es distinta según el rango de edad debido a diferentes actitudes, estilos de vida y opiniones $(11,18,32)$. Sriphadungporn et al. (11) encontraron que la edad tiene un impacto en la percepción de la sonrisa en relación a la exposición gingival y triángulos negros, pero no en cuanto a la posición del borde incisal de ICS cuando comparó personas comunes, un grupo joven de 15 a 29 años y otro adulto de 36 a 52 años, por lo cual se infiere en cuanto a esta variable que la edad no sería un sesgo para nuestro estudio que presenta como promedio una población adulto joven; sin embargo debido a que en el antecedente previo de angulación mesiodistal 
del ICS (23) y en nuestra investigación, la edad no ha sido un factor de estudio se sugiere sea considerado para futuras investigaciones.

Nuestro análisis de datos para la posición vertical del ICS nos muestra que las personas comunes solo dieron una mayor calificación que los estudiantes de odontología con diferencias estadísticamente significativas solamente en la categoría de $1 \mathrm{~mm}$, resultados que se oponen parcialmente a lo reportado en estudios previos $(3,33)$ en donde se encontró que los dentistas son más críticos que las personas comunes y dan puntajes menores en la mayoría de los casos. En nuestro estudio no se estaría encontrando esta diferencia, posiblemente porque los estudiantes de odontología son un grupo que aún se encuentra en formación académica y no tiene definido por completo los conceptos de sonrisa estética. La posición vertical del ICS de 1 y $2 \mathrm{~mm}$ obtuvieron la mejor puntuación para estudiantes de odontología, y la de $1 \mathrm{~mm}$ para personas comunes, siendo esta preferencia coincidente con lo reportado por Simões et al. (21) y similar al valor más estético de $1.5 \mathrm{~mm}$ encontrado por Machado et al. (8) En contraste, King et al. (34) reportaron la mejor puntuación para una distancia de $0.6 \mathrm{~mm}$ entre el borde incisal del ICS e ILS, debido a esta variabilidad se sugiere una adecuada comunicación entre especialistas, odontólogos generales y pacientes durante la planificación del tratamiento.

Al evaluar la angulación mesiodistal del ICS las personas comunes dieron una mayor calificación que los estudiantes de odontología en las tres posiciones $\left(-6^{\circ}, 0^{\circ}\right.$ y $\left.4^{\circ}\right)$, en contraste a lo reportado por Yang et al. (23) quienes encontraron que la profesión no altera la percepción cuando evaluaron fotografías de hombre y de mujer, del tercio inferior y de toda la cara; a diferencia de nuestro estudio en donde solo se evaluó una fotografía del tercio inferior de mujer. Por otro lado, fue coincidente en que la categoría de $0^{\circ}$ considerada la ideal obtuviera la calificación más alta.

Al comparar la percepción por región se encuentra que en la mayoría de los casos los resultados obtenidos coinciden con lo mencionado en la literatura $(11,13)$ en donde la percepción varía según el país, región o continente. Las diferencias encontradas entre los habitantes de la región costa, sierra y selva podría atribuirse a las diferencias sociales, económicas, culturales y de origen étnico; lo cual estaría influenciando en sus expectativas de la estética de la sonrisa.

Los encuestados de la región costa (Lima) calificaron con menores puntuaciones en la mayoría de los casos, esto podría deberse a que es la ciudad capital y tiene en general un mayor desarrollo económico y social, además en el caso de los estudiantes de odontología, la universidad a la que pertenecían era particular y se ubicaba en el distrito de Miraflores de mayor auge económico y nivel educativo considerado de clase media alta; lo cual coincide con el estudio de Dindaroglu (14) quien refiere que el nivel de educación es un factor sociodemográfico que influye en la percepción de la sonrisa, disminuyendo las calificaciones mientras más alto sea el nivel.

El presente trabajo ha sido realizado en una población de un contexto específico por lo cual la generalización se podrá realizar a poblaciones con características similares. Asimismo, los resultados encontrados sobre la percepción estética de la sonrisa en cuanto al ICS podrían ser utilizados en las diferentes regiones peruanas como parámetro clínico para lograr una adecuada finalización y acabado de los tratamientos de ortodoncia, así como referencia de la percepción en los casos de recidiva dentaria, teniendo en cuenta que el tratamiento debe ser individualizado con una adecuada comunicación entre los profesionales y pacientes. 


\section{CONCLUSIONES}

La percepción estética de la sonrisa es afectada por la variación de posición vertical y angulación del ICS en personas comunes y estudiantes de odontología en las tres regiones del Perú, siendo los estudiantes de odontología los que califican con menor puntaje.

\section{CONFLICTO DE INTERESES}

Los autores declaramos no tener ningún tipo de conflicto de interés.

\section{AGRADECIMIENTOS}

Al Técnico en Computación e Informática Alexander Martín García Gonzales y a la Especialista en Ortodoncia y Ortopedia Maxilar Itala Paulita Flores Concha por su apoyo logístico y contribución en la toma y edición de las imágenes.

\section{REFERENCIAS}

1. Al Taki A., Khalesi M., Shagmani M., Yahia I., Kaddah F. A. Perceptions of altered smile esthetics: a comparative evaluation in orthodontists, dentists, and laypersons. Int J Dent. [Internet].2016 [acceso 8 de Octubre 2018]; 2016: 1-11. Disponible en: http:// dx.doi.org/10.1155/2016/7815274

2. Alhammadi M. S., Halboub E., Al-Mashraqi A. A., Al-Homoud M., Wafi S., Zakari A., Mashali W. Perception of facial, dental, and smile esthetics by dental students. J Esthet Restor Dent. 2018; 30 (5): 415-426.

3. An S. M., Choi S. Y., Chung Y. W., Jang T. H., Kang K. H. Comparing esthetic smile perceptions among laypersons with and without orthodontic treatment experience and dentists. Korean J Orthod. 2014; 44 (6): 294-303.

4. Öz A. A., Akdeniz B. S., Canlı E., Çelik S. Smile Attractiveness: Differences among the Perceptions of Dental professionals and Laypersons. Turk J Orthod. 2017; 30 (2): 50-55.

5. Correa B. D., Bittencourt M. A. V., Machado AW. Influence of maxillary canine gingival margin asymmetries on the perception of smile esthetics among orthodontists and laypersons. Am J Orthod Dentofacial Orthop. 2014; 145 (1): 55-63.

6. Peck S., Peck L. Selected aspects of the art and science of facial esthetics. Semin Orthod. 1995; 1 (2): 105-26.

7. Machado A.W. 10 commandments of smile esthetics. Dental Press J Orthod. 2014; 19 (4): 136-57.

8. Machado A. W., McComb R. W., Moon W., Gandini L. G. Jr. Influence of the vertical position of maxillary central incisors on the perception of smile esthetics among orthodontists and laypersons. J Esthet Restor Dent 2013; 25 (6): 392-401.

9. Betrine Ribeiro J., Figuereido B. A., Machado A.W. Does the presence of unilateral maxillary incisor edge asymmetries influence the perception of smile esthetics? J Esthet Restor Dent. 2017; 29 (4): 291-297.

10. Cheng H. C., Cheng P. C. Factors affecting smile esthetics in adults with different types of anterior overjet malocclusion. Korean J Orthod. 2017; 47 (1): 31-38.

11. Sriphadungporn C., Chamnannidiadha N. Perception of smile esthetics by laypeople of different ages. Progress in Orthodontics. 2017; 18 (1): 8.

12. Ayyildiz E., Tan E., Keklik H., Celebi A. A., Pithon M. M. Evaluation of black spaces between maxillary central incisors by dentistry students and laypeople. J Oral Sci. 2017; 59 (3): 323-28.

13. Ioi H., Kang S., Shimomura T., Kim S. S., Park S. B., Son W. S., et al. Effects of vertical positions of anterior teeth on smile esthetics in Japanese and Korean orthodontists and orthodontic patients. J Esthet Restor Dent. 2013; 25 (4): 274-82. 
14. Dindaroğlu F., Özmutlu M. K., Işıksal E. The effect of educational status on the perception of social and spontaneous smiles. Turk $\mathrm{J}$ Orthod 2016; 29 (1): 10-5.

15. Russello $\mathrm{S}$. The impact of media exposure on self-esteem and body satisfaction in men and women. J Interdisciplinary Undergrad Res. 2013; 1 (1): 1-12.

16. Parrini S., Rossini G., Castroflorio T., Fortini A., Deregibus A., Debemardi C. Laypeople's perceptions of frontal smile esthetics: A systematic review. Am J Orthod Dentofacial Orthop. 2016; 150 (5): 740-750.

17. NandaR. EstéticayBiomecánica en Ortodoncia. 2da ed. Venezuela: Amolca Actualizaciones médicas, 2017.

18. Mokhtar H. A., Abuljadayel L. W., Al-Ali R. M., Yousef M. The perception of smile attractiveness among Saudi population. Clin Cosmet Investig Dent. 2015; 20 (7): 17-23.

19. Pitts T. R. Bracket Positioning for Smile Arc Protection. J Clinic Orthod. 2017; 51 (3), 142-56.

20. Cabral Menezes E. B., Viera Bittencourt M. A., Machado A.W. ¿Do different vertical positions of maxillary central incisors influence smile esthetics perception? Dental Press J Orthod. 2017; 22 (2): 95-105.

21. Simões D., Meyge de Brito G., Teixeira Cangussu M. C., Machado A. W. Does the vertical position of maxillary central incisors in men influence smile esthetics perception? Am J Orthod Dentofacial Orthop. 2019; 156 (4): 485-492.

22. Machado R. M., Assad Duarte M. E., Jardim da Motta A. F., Mucha J. N., Motta A. T. Variations between maxillary central and lateral incisal edges and smile attractiveness. Am J. Orthod Dentofacial Orthop. 2016; 150 (3): 425-35.
23. Yang S., Guo Y., Yang X., Zhan F., Wang J., Qiu J., Li J. Effect of mesiodistal angulation of the maxillary central incisors on esthetic perception of the smile in the frontal view. Am J Orthod Dentofacial Orthop 2015; 148 (3): 396-404.

24. Rayyan M.R. Effect of the interproximal contact level on the perception of smile esthetics. Dent Med Probl. 2019; 56 (3): 251-255.

25. De Almeida-Pedrin R. R., Pinzan A., de Almeida R. R., Ursi W., de Almeida M. R. Panoramic evaluation of mesiodistal axial inclinations of maxillary anterior teeth in orthodontically treated subjects. Am J Orthod Dentofacial Orthop 2006; 130 (1): 56-60.

26. Bass N. M. Measurement of the profile angle and the esthetic analysis of the facial profile. J Orthod 2003; 30 (1): 3-9.

27. Adams M., Andrews W., Tremont T., Martin C., Razmus T., Gunel E., Ngan P. Anteroposterior relationship of the maxillary central incisors to the forehead in adult white males. Orthodontics (Chic.). 2013; 14 (1): 2-9.

28. Malquichagua-Veramendi C., Liñán-Durán C., Ríos-Villasis K. Percepción estética de la sonrisa con respecto a la inclinación del incisivo central superior. Rev. Estomatol. Herediana. 2014; 24 (3): 155-162.

29. Almutairi T. K., Albarakati S. F., Aldrees A. M. Influence of bimaxillary protrusión on the perception of smile esthetics. Saudi Med J. 2015; 36 (1): 87-93.

30. Howells D. J., Shaw W. C. The validity and reliability of ratings of dental and facial attractiveness for epidemiologic use. Am J Orthod. 1985; 88 (5): 402-8.

31. Kaya B., Uyar R. Influence on smile attractiveness of the smile arc in conjunction with gingival display. Am J Orthod Dentofacial Orthop. 2013; 144 (4): 541-7. 
32. Laceda-Santos R., Pereira T. B., Pithon M. M. Esthetic perception of the buccal corridor in different in different facial types by laypersons of different ages. Biosci J. 2015; 31 (4): 1283-90.

33. Ousehal L.,Aghoutan H., Chemlali S., Anssari I. F., Talic N. Perception of altered smile esthetics among Moroccan professionals and lay people. Saudi Dental Journal. 2016; 28 (4): 174-182.

34. King K. L., Evans C. A., Viana G., Begoles E., Obrez A. Preferences for vertical position of the maxillary lateral incisors. World $\mathrm{J}$ Orthod. 2008; Summer: 9 (2): 147-54. 